\title{
MS07-01 | Taking Crystallographic Snapshots of Vitamin B6 Biosynthesis and Treating Specific Radiation Damage
}

Tews, Ivo (University of Southampton, Southampton, GBR)

The enzyme complex PLP synthase catalyses a complex sequence of chemical reactions in the biosynthesis of vitamin B6. Structures of covalent enzyme-intermediate complexes illuminate the reaction cascade, starting from simple triose and pentose carbohydrates and the amino acid glutamine, and ending in formation of the aromatic heterocycle PLP, an active form of vitamin B6.

The main catalyst for this reaction is the Pdx1 enzyme which possesses a TIM-like $(\beta \alpha)_{8}$-barrel fold. Together with the Pdx2 glutaminase, Pdx1 forms the PLP synthase complex with a molecular weight of 750 kDa. The Pdx1 dualspecificity active site binds pentose and triose carbohydrates in sequence. The final product PLP, however, is observed in a second binding site.

An intermediate with a characteristic absorbance is key to understand the sophisticated enzyme structure. This intermediate is formed from the pentose ribose 5-phosphate and ammonia and bridges across binding sites by double Schiff base formation. A lysine relay mechanism to migrate carbonyl intermediates provides an elegant solution to the challenge of coordinating a complex sequence of reactions that follow a path of over $20 \AA$ between substrate- and product-binding sites.

Electron rich compounds such as these intermediates are prone to specific radiation damage that can lead to misinterpretation. Dose-conservative diffraction analysis can limit the effects of radiation induced damage. Radiation induced decay was monitored using the spectroscopic signature of the intermediates, detected in crystallo using on-line micro-spectrophotometry at the time of crystallographic data collection. Low-dose multicrystal data support the bridging structure of the intermediate. 